\title{
Does cognitive behavior therapy alter emotion regulation in inpatients with a depressive disorder?
}

This article was published in the following Dove Press journal:

Psychology Research and Behavior Management

12 May 2014

Number of times this article has been viewed

\author{
Thomas Forkmann' \\ Anne Scherer' \\ Markus Pawelzik ${ }^{2}$ \\ Verena Mainz' \\ Barbara Drueke' \\ Maren Boecker' \\ Siegfried Gauggel' \\ 'Institute of Medical Psychology \\ and Medical Sociology, University \\ Hospital of RWTH Aachen, Aachen, \\ Germany; ${ }^{2}$ EOS Hospital for \\ Psychotherapy, Hammer Münster, \\ Germany
}

\begin{abstract}
Introduction: Emotion regulation plays an important role in the development and treatment of depression. The present study investigated whether the emotion regulation strategies, expressive suppression (ES) and cognitive reappraisal (CR) change in the course of cognitive behavior therapy (CBT) of depressive inpatients. Furthermore, it also examined whether changes in $\mathrm{CR}$ and ES correlated with positive treatment outcomes.
\end{abstract}

Methods: Forty-four inpatients from a psychotherapeutic hospital who suffered from a depressive disorder ( mean age $=36.4$ years, standard deviation $=13.4$ years; $63.6 \%$ female) filled in the Emotion Regulation Questionnaire and the Beck Depression Inventory at admission and discharge. To detect changes in emotion regulation, and depression across treatment, data were analyzed using multivariate analyses of variance (MANOVA) for repeated measures, effect sizes, and Spearman correlations. A $P$-value of $\leq 0.05$ was considered statistically significant.

Results: Depression severity $\left(F[1]=10.42, P=0.003 ; \eta^{2}=0.22\right)$ and $\mathrm{CR}(F[1]=4.71, P=0.04$; $\left.\eta^{2}=0.11\right)$ changed significantly across CBT treatment. ES remained virtually stable. Post-treatment scores of CR were also positively correlated with reduction in depressive symptoms across treatment $(\rho=0.30, P=0.05)$.

Conclusion: The results suggest that $\mathrm{CBT}$ affects emotion regulation in depressive inpatients only for $\mathrm{CR}$ and that higher post-treatment scores in $\mathrm{CR}$ were related to greater reduction in depressive symptoms across treatment.

Keywords: emotion regulation, depression, major depressive disorder, psychotherapy

\section{Introduction}

In our everyday life, things happen that make people feel sad, angry, or confused and which can cause negative emotional states. As such, a negative emotional state is aversive, most people try to overcome negative emotions by using a variety of behavioral and/or cognitive strategies. The process to alter these states is usually called emotion regulation. Researchers' interest in emotion regulation has grown over the past two decades. In the early 1990 s only a few papers on emotion regulation were published, but now there are many thousands each year. ${ }^{1,2}$ New statistical approaches such as mediation analysis were applied and new techniques developed (eg, functional magnetic resonance imaging or experience sampling methods) so that much progress in the understanding of the function, structure, and individual differences in emotion regulation could be achieved. ${ }^{3}$

Emotion regulation has been conceptualized as "processes through which individuals modulate their emotions consciously and subconsciously to respond to environmental demands". ${ }^{4}$ A multitude of different emotion regulation strategies has been proposed.
Correspondence: Thomas Forkmann, Institute of Medical Psychology and Medical Sociology, University Hospital of RWTH Aachen University, Pauwelsstr 19, Aachen 52074, Germany Tel +492418089003

Fax +49 24I 803389003

Email tforkmann@ukaachen.de 
Some of them, such as reappraisal and problem solving, have been seen as adaptive, whereas others, such as suppression and avoidance, have been seen as maladaptive strategies. ${ }^{4}$ Many contemporary models of psychopathology incorporate emotional regulation deficits or emotion misregulation (for an overview see Aldao et $\mathrm{al}^{4}{ }^{4}$ and Berking and Wupperman ${ }^{5}$ ). One of the most extensively investigated models of emotion regulation has been presented in the seminal work of Gross et al and Gross. ${ }^{6,7}$ They formulated a process model of emotion regulation and postulated antecedent- and responsefocused emotion regulation strategies. Most subsequent research on that model focused on the emotion regulation strategies cognitive reappraisal (CR) (an antecedent-focused strategy) and expressive suppression (a response-focused strategy).

According to Lazarus and Alfert, ${ }^{8} \mathrm{CR}$ means a form of cognitive change that involves constructing a potentially emotion-eliciting situation in a way that changes its emotional impact. Suppression on the other hand involves inhibiting ongoing emotion-expressive behavior. ${ }^{7} \mathrm{CR}$ occurs early in the emotion generation process and thus has the ability to alter the whole process, involving the experience and expression of emotion. It should therefore be capable of successfully decreasing the experience and overt expression of negative emotion. Suppression, on the other hand, targets the overt behavioral expression of emotion only. Therefore, it is not supposed to down-regulate the experience of negative emotion which may thus continue to remain unresolved.

Some predictions of this model have been tested experimentally. By and large, the results support the validity of the model. It has been shown that people instructed to suppress their emotions showed less expressive behavior but experienced as much negative emotion as participants who did not intentionally regulate their emotion, whereas reappraisal decreased both the experience and behavioral expression of negative emotion. ${ }^{7}$ In another experiment, suppression but not reappraisal led to memory impairment for social information presented while the individual was regulating emotions. ${ }^{9}$ Additionally, suppression has been found to exert the potential to undermine social functioning to a much greater extent than reappraisal by disrupting emotional communication. ${ }^{10}$ Sheppes et $\mathrm{al}^{11}$ showed that disengagement with emotional processing (such as distraction) is more effective in down-regulating negative emotion than reappraisal when emotion intensity is high, whereas reappraisal is more effective when emotion intensity is rather low. Moreover, they reported results of three experiments that all showed a relative preference of participants to choose disengagement when negative emotion intensity is high and reappraisal when negative emotion intensity is low. ${ }^{11,12}$

From early on, individual differences in suppression and reappraisal have also been linked to various aspects of personal well-being. Gross and $\mathrm{John}^{6}$ reported a set of studies which demonstrated that reappraisers as compared to suppressers have fewer depressive symptoms, greater self-esteem, life satisfaction, and general well-being. These findings suggest that there is a link between individual differences in these two strategies and the development and maintenance of depressive disorders. Other studies supported the claim that more frequent use of certain strategies (eg, expressive suppression [ES]) and less frequent use of other strategies (eg, CR) are related to heightened levels of mental symptoms burden. ${ }^{6,13-16}$

The recent meta-analysis by Aldao et $\mathrm{al}^{4}$ showed that low levels of reappraisal and high levels of suppression were linked to high levels of depression. For suppression, this relation is even stronger in adult samples than in samples of children or adolescents. Four studies examined the impact of suppression on depression longitudinally. In three samples college students' scores in suppression predicted depressive symptoms at 7 weeks, ${ }^{17} 10$ weeks, ${ }^{18}$ and 6 months later. ${ }^{19}$ In another study suppression interacted with social anxiety to predict the effect over 3 months. ${ }^{20}$ Despite this strong link between emotion regulation and mental symptom burden, especially depression, there is a clear lack of studies that can examine the role of emotion regulation as operationalized in the Grossian model ${ }^{6}$ in cognitive behavior therapy (CBT) for depression. This is surprising since evidence suggests that emotion-regulation skills predict status of mental health at later points in time. ${ }^{21}$ Berking et $\mathrm{al}^{22}$ presented data suggesting that CBT changed emotion regulation in a mixed sample of patients with mental disorders. In addition, they could show slightly larger treatment gains for patients who received specific training in emotion regulation skills in addition to CBT as compared to patients who received only CBT-based treatment as usual. However, analyses have not been broken down by diagnostic groups; therefore no definite conclusions could be drawn that were specific for depression.

Taken together, there is extensive evidence suggesting that emotion regulation may play an important role in the development and maintenance of mental disorders. Especially for depression, it has been shown that more use of ES and less use of CR is associated with greater depressive symptom burden. However, only small amounts of data are available so far on the role of emotion regulation in $\mathrm{CBT}$ for depression that does 
not explicitly target emotion regulation. Thus, it is unclear whether under these circumstances successful treatment goes hand in hand with changes in emotion regulation.

Therefore, the present pilot study investigated whether the emotion regulation strategies ES and CR change in the course of treatment of depressive inpatients undergoing CBT and whether such change correlates with positive treatment outcome. In line with previous research, we hypothesized that patients suffering from a depressive disorder should show relatively high levels of suppression and low levels of CR before treatment. We further hypothesized that successful treatment outcome is related to increased use of CR and decreased use of suppression.

\section{Material and methods}

\section{Participants}

The sample consisted of 44 consecutive inpatients of a German psychotherapeutic hospital who were suffering from a depressive disorder according to the criteria of the of the International Classification of Diseases 10th edition (ICD-10). ${ }^{23}$ All patients approached agreed to participate. The age of the participants ranged from 18 to 66 years, with a mean age of 36.4 years (standard deviation $[\mathrm{SD}]=13.4$ years). Twenty-eight (63.6\%) patients were female. Mean duration of stay in hospital was 59 days ( $\mathrm{SD}=39$ days). Comorbid diagnoses included eating disorders $(31.8 \%)$, anxiety disorders $(15.9 \%)$, and personality disorders $(15.9 \%)$. The hospital specializes in treatment of affective and eating disorders, which accounts for the high frequency of eating disorders in the present sample. Diagnoses were verified in a twostep procedure: first, patients were assessed by the treating therapist using a clinical interview in which the International Diagnostic Checklists (IDCLs) were applied. ${ }^{24}$ The IDCLs are checklists that can be used to make a careful evaluation of the symptoms and classification criteria, and thus help to arrive at precise diagnoses according to ICD-10. In the second step, diagnoses were verified through clinical conferences including senior psychotherapists and psychiatrists. All patients gave informed consent and participated voluntarily. None of the participants was paid. Study procedures were in accordance to the Declaration of Helsinki.

The treatment was administered in one-to-one meetings by psychotherapists in training or charted psychotherapists all extensively trained in CBT. Psychotherapists in training were supervised once weekly by a senior psychotherapist to ensure adherence to the treatment. Main interventions consisted of behavioral activation, cognitive restructuring, and skills training. ${ }^{3,25}$ Based on the individual biography and functional analysis of depressive behaviors, the patient is guided to actively overcome deficits in activity rate, beliefs, and interpersonal skills. In addition to the one-to-one sessions, group therapy settings with video feedback focused on interpersonal skills training.

The therapeutic input is comparably high: each patient received on average 2 hours of individual therapy and 4 hours of group therapy per working day. Specific interventions that focus on emotion regulation abilities were not part of the treatment program. No participants dropped out of treatment and no severe treatment complications occurred.

\section{Instruments}

\section{Beck Depression Inventory}

The Beck Depression Inventory (BDI) was used to provide a quantitative measure of depression. ${ }^{26,27}$ It contained 21 items with item scores ranging from 0 to 3 . Participants were asked to choose one or more statements per item that best represented their mental state during the last week. A total score of $\geq 11$ indicates mild to moderate depression and a total score of $\geq 18$ indicates moderate to severe depression. Reliability (Cronbach's $\alpha$ ) of the BDI in the present sample was $\alpha=0.86$ at admission and $\alpha=0.89$ at discharge.

\section{Emotion Regulation Questionnaire}

To detect the individual differences in the habitual use of the emotion regulation strategies cognitive reappraisal and expressive suppression, all patients filled in the Emotion Regulation Questionnaire (ERQ). ${ }^{6}$ The ERQ consists of ten items. Six of them constitute the scale "cognitive reappraisal" and four constitute the scale "expressive suppression". Answer options range from 1 "strongly disagree" to 7 "strongly agree". Maximum score is 42 for CR and 28 for ES indicating high levels of the respective emotion regulation strategy. Reliability (Cronbach's $\alpha$ ) of the scale $\mathrm{CR}$ in the present sample was $\alpha=0.73$ at admission and $\alpha=0.55$ at discharge. Inspection of item characteristics of all items of the CR scale revealed insufficient separation for item five at both time points (admission: 0.14; discharge: -0.11 ). We then inspected the item wordings and suspected that the German translation of item five may be slightly misleading. It was therefore decided to exclude this item from the scale for the present analyses which led Cronbach's $\alpha$ to rise to $\alpha=0.78$ on admission and to $\alpha=0.69$ on discharge. Reliability (Cronbach's $\alpha$ ) of the scale ES in the present sample was $\alpha=0.78$ at admission and $\alpha=0.90$ at discharge.

All participants completed the BDI, the ERQ, and a demographical data sheet at admission and the BDI and the ERQ at discharge. 
Each test session started with the diagnostic interview. Additional clinical data were taken from medical records.

\section{Data analysis}

Effect of CBT on emotion regulation and depression

In order to evaluate the effect of the CBT treatment on depressive symptoms and emotion regulation, multivariate analyses of variance for repeated measures (MANOVA) were conducted with time (pre- versus post-treatment scores) as independent within-subjects variable and depression, ES and $\mathrm{CR}$ as dependent variables. Since age and sex have been shown to be associated with different types of emotion regulation, these variables were entered as covariates. ${ }^{28}$ Prior to MANOVA, homogeneity of error variances was tested with the Mauchly test ${ }^{29}$ for sphericity. Homogeneity of error variances is important in order to interpret the results of MANOVA validly.

Therefore, in case of significant results of the Mauchly test and thus violation of homogeneity of error variances, a Greenhouse-Geisser ${ }^{30}$ correction was conducted. In addition, the partial $\eta^{2}$ was calculated as effect size. According to Cohen ${ }^{31,32}$ a partial $\eta^{2}$ of 0.01 can be viewed as small, 0.06 as medium, and 0.16 as large effect.

\section{Relationship between emotion regulation and mental health across treatment}

Spearman's correlations were used to assess univariate associations between pre-treatment scores on ES and CR and pre-treatment scores on BDI, between pre-treatment scores on ES and CR and change in BDI and between change in scores on ES and CR and change in BDI. All analyses were performed using SPSS 17 for Windows (IBM Corporation, Armonk, NY, USA).

\section{Results}

\section{Effect of CBT on emotion regulation}

\section{and depressive symptoms}

MANOVA revealed a significant multivariate effect of time on measures for depression, CR, and $\operatorname{ES}(F[3,39]=4.98$, $P=0.005$; Wilks' $\lambda=0.71$; partial $\eta^{2}=0.29$ ). No interaction effect was found with age or sex. Table 1 shows univariate comparisons. A significant change across treatment was found for depression $\left(F[1]=10.42, P=0.003 ; \eta^{2}=0.22\right)$ with lower scores after treatment than at admission and $\mathrm{CR}\left(F[1]=4.71, P=0.04 ; \eta^{2}=0.11\right)$ showing higher scores after treatment as compared to admission. ES showed virtually no change across treatment $(F[1]=0.002, P=0.97$; $\left.\eta^{2}<0.001\right)$.
Table I Results of MANOVA pre- and post-treatment scores of ES, $\mathrm{CR}$, and $\mathrm{BDI}$; outcome measures and corresponding effect sizes

\begin{tabular}{|c|c|c|c|c|c|c|c|}
\hline & \multicolumn{2}{|c|}{$\begin{array}{l}\text { Pre- } \\
\text { treament }\end{array}$} & \multicolumn{2}{|c|}{$\begin{array}{l}\text { Post- } \\
\text { treatment }\end{array}$} & \multirow[t]{2}{*}{$F$} & \multirow[t]{2}{*}{$P$} & \multirow[t]{2}{*}{$\eta^{2}$} \\
\hline & $\overline{\mathbf{M}}$ & $\overline{\text { SD }}$ & $\bar{M}$ & $\overline{S D}$ & & & \\
\hline$\overline{\mathrm{ES}}$ & 14.9 & 5.8 & 13.6 & 5.3 & 0.002 & 0.97 & $<0.001$ \\
\hline$C R$ & 18.1 & 5.7 & 22.2 & 4.4 & 4.71 & 0.04 & 0.11 \\
\hline BDI & 22.4 & 9.7 & 13.5 & 9.1 & 10.42 & $<0.01$ & 0.22 \\
\hline
\end{tabular}

Notes: $P$, empirical significance; $\eta^{2}$, effect size. Figures in bold are significant at $P \leq 0.05$.

Abbreviations: MANOVA, multivariate analyses of variance; ES, expressive suppression; CR, cognitive reappraisal; SD, standard deviation; M, mean; BDI, Beck Depression Inventory.

Overall, successful treatment in terms of remission (posttreatment BDI total score $<11)$ was observed for $26(59.1 \%)$ out of all 44 patients.

\section{Relationship between emotion regulation and mental health across treatment}

Emotion regulation was associated with pre-treatment depression status for the strategy of ES (pre-treatment scores of ES: $\rho=0.34 ; P=0.02$; post-treatment scores of ES: $\rho=0.39 ; P=0.01$ ) but not for CR. Thus, higher values in ES were associated with more severe depression as indicated by higher scores in the BDI. Post-treatment scores of CR were correlated with symptom change ( $\rho=0.30, P=0.05$ ). This means that those patients who reported higher levels of $\mathrm{CR}$ after treatment also experienced larger relief in symptom burden (Table 2). No further significant correlations were found.

\section{Discussion}

The purpose of the present study was to investigate whether the emotion regulation strategies ES and CR change in the course of treatment in inpatients suffering from a depressive disorder undergoing CBT and whether such change correlates with positive treatment outcomes. We found significant treatment effects, which were large as indicated by the measure of effect size $\eta^{2}$ for CR and depression. There was virtually no effect of treatment on ES.

Previous research suggested that high scores on ES and low scores on CR are associated with depression in both clinical and non-clinical samples; we expected to find a decrease of ES and an increase of CR due to CBT. ${ }^{6,4,17}$ However, change of emotion regulation was only found for CR while ES remained virtually stable. Pre- and post-treatment assessment of ES was positively correlated with pre-treatment assessments of depression. Furthermore, post-treatment scores of CR were positively correlated with depressive symptom change. Thus, those patients who reported higher levels of CR after treatment also experienced larger relief in 
Table 2 Correlations between pre- and post-treatment scores and change scores of ES and CR and depressive symptoms

\begin{tabular}{|c|c|c|c|c|c|c|c|c|c|c|c|c|}
\hline & \multicolumn{4}{|c|}{ Pre-treatment } & \multicolumn{4}{|c|}{ Post-treatment } & \multicolumn{4}{|c|}{ Change } \\
\hline & \multicolumn{2}{|l|}{$\overline{C R}$} & \multicolumn{2}{|l|}{ ES } & \multicolumn{2}{|l|}{$\overline{C R}$} & \multicolumn{2}{|l|}{ ES } & \multicolumn{2}{|l|}{$\overline{C R}$} & \multicolumn{2}{|l|}{ ES } \\
\hline & $\rho$ & $P$ & $\rho$ & $P$ & $\rho$ & $P$ & $\rho$ & $P$ & $\rho$ & $P$ & $\rho$ & $P$ \\
\hline \multicolumn{13}{|l|}{$\mathrm{BDI}$} \\
\hline Pre & -0.10 & 0.55 & 0.34 & 0.02 & 0.11 & 0.48 & 0.39 & 0.01 & -0.13 & 0.43 & -0.04 & 0.78 \\
\hline Post & -0.02 & 0.93 & 0.05 & 0.75 & -0.20 & 0.20 & 0.27 & 0.08 & 0.01 & 0.95 & -0.22 & 0.15 \\
\hline Change & -0.04 & 0.80 & 0.26 & 0.09 & 0.30 & 0.05 & 0.05 & 0.74 & -0.10 & 0.56 & 0.24 & 0.11 \\
\hline
\end{tabular}

Notes: $\rho$, Spearman's correlation coefficient; $P$, significance; Figures in bold are significant at $P \leq 0.05$.

Abbreviations: ES, expressive suppression; CR, cognitive reappraisal; BDI, Beck Depression Inventory.

symptom burden. Consequently, this result may be interpreted as indicating that $\mathrm{CBT}$ results in significant increases in $\mathrm{CR}$ and that higher scores in CR after CBT are correlated with greater change in depressive symptoms.

Obviously, classical CBT for depression in an everyday setting did not affect the extent to which patients use ES to regulate their emotions in the present study. Simultaneously, CR was changed significantly across treatment. One explanation for this discrepancy could be that ES is basically a strategy targeting the expression of overt behavior while $\mathrm{CR}$ is a cognitive strategy targeting the individual appraisal of situations, thoughts and feelings. Although change of cognition in treatment is usually more difficult and takes more time than change of behavior, the cognitive restructuring, ie, development of new, more functional appraisements of individual experiences, is a central element of CBT for any mental disorder whereas the training of overt emotional expression might be less common in CBT. Consequently, greater effects of $\mathrm{CBT}$ on cognitive rather than behavioral emotion regulation strategies appear logical.,

The fact that only CR but not emotional suppression changed across treatment could also be interpreted as indicating an effect inherent to classical CBT which traditionally focuses more directly on cognition than on emotion. More precisely, the focus in classical CBT is on concrete dysfunctional thoughts and the alteration of their contents (eg, "I am a complete failure") rather than on emotional or (meta-) cognitive processes. Only recently, evidence has been presented indicating that the change of metacognitive processes particularly, could be an important treatment target in CBT for depression. ${ }^{33-35}$ For example, Fresco et al ${ }^{34}$ showed that the ability to take a "decentered" perspective on one's own thoughts conferred protection from depressive relapse. Decentering has been defined as the ability to observe one's own thoughts and feelings as temporary, objective events in the mind, as opposed to reflections of the self that are necessarily true, and is thus a metacognitive process. ${ }^{36}$ The relatively newly developed mindfulnessbased cognitive therapy (MBCT) focuses on the function of metacognition on mental health and proposes positive and long-lasting effects of metacognitive changes especially for patients formerly suffering from a depressive disorder. ${ }^{37-40}$ It might be speculated that MBCT may have a more pronounced effect on ES than classical CBT because MBCT conveys a more decentered perspective on one's own emotional processing and emotional expressive behavior. Thus, it may be an interesting task for future research to investigate whether MBCT not only produces a reduced risk for relapse but also changes utilization of ES in formerly depressed patients and whether this is mediated by patients' acquisition of a more decentered perspective on their own thoughts and feelings.

\section{Limitations}

Some limitations of the present study have to be taken into account when interpreting the results. First, sample size was small; therefore larger sample sizes are needed to provide further support for the reported results. Thus, the present study may be seen as a pilot study that can stimulate a discussion on the research question raised, and additional data is needed to further shed light on this issue. Second, no follow-up examination was conducted so nothing can be said about the stability of the presented findings or potential further increases of effects on emotion regulation in subsequent outpatient treatment. Third, the study was conducted in an everyday naturalistic setting of inpatient psychotherapy. This might have been advantageous with regards to ecological validity; changes observed in the study are likely to represent features of everyday routine care in a psychotherapeutic hospital and are not limited to some laboratory setting. However, simultaneously, a control group was lacking. Thus, it cannot be ruled out that any psychotherapeutic intervention would have impacted emotion regulation, ie, the changes are not unique to CBT, or that nonspecific factors may have accounted for at least 
part of the presented findings. Fourth, we cannot rule out the possibility that other features of inpatient treatment besides CBT could have accounted for the changes that were observed in emotion regulation in the present study that might limit the validity of the presented results. Fifth, no detailed information about duration of illness, functional status of inpatients and subsidiary antidepressant treatment were available. Sixth, when appreciating the current results one has to keep in mind that item five of the ERQ was excluded from sum score calculation of the CR scale due to very low item separation. This led to substantially improved internal reliability of the $\mathrm{CR}$ scale. A possible reason for the insufficient item separation may be a misleading item content of our German translation of this item. The wording of item five has to be checked in future studies to increase its item separation value. Seventh, it has to be noted that the criterion for discharge from the psychotherapeutic clinic was not recovery but rather the evidence that the mental illness and symptom burden had receded sufficiently so that the patient was able to successfully manage his or her everyday life and attend a subsequent outpatient psychotherapeutic treatment. Therefore, it seems that the effect of treatment on self-reported depression was clinically sufficient to warrant discharge but might further increase in subsequent outpatient treatment. Eighth, it appears that at time of discharge, cognitive changes might have already been achieved but changes in overt behavioral expression of emotion as indicated by changed ES might not develop until patients have progressed in subsequent ambulatory treatment. Finally, emotion regulation was measured only with regard to ES and CR. However, the issue of how many dimensions of emotion regulation could be meaningfully differentiated in self-report instruments is still subject to vivid discussions and some authors have presented instruments measuring different types of emotion regulation strategies. ${ }^{41,14}$ Thus, it could be that more pronounced effects of classical CBT on other emotion regulation strategies that were not assessed in the present investigation can be found.

\section{Conclusion}

We found evidence that classical CBT for depression that did not explicitly target emotion regulation resulted in changes of a subtype of emotion regulation in depressive inpatients in an everyday, naturalistic setting. While patients showed higher self-reported CR after treatment, ES remained stable. Higher post-treatment scores of cognitive reappraisal were also positively related with greater reduction in depressive symptoms across treatment. Possible reasons for these results and potential study limitations have been discussed. Future studies should include at least one follow up assessment of patients in order to provide data that could offer more information on long-term effects of changes in emotion regulation in the course of CBT, and it might be interesting to compare the effects of classical CBT and MBCT on patients' emotion regulation styles.

\section{Disclosure}

The authors have no conflicts of interest to disclose in this work.

\section{References}

1. Koole S. The psychology of emotion regulation: an integrative review. Cognition and Emotion. 2009;23:4-41.

2. Gross JJ. Emotion regulation: taking stock and moving forward. Emotion. 2013;13(3):359-365.

3. Gilson M, Freeman A, Yates MJ. Overcoming Depression: A Cognitive Therapy Approach, Therapist Guide. New York: Oxford University Press; 2009.

4. Aldao A, Nolen-Hoeksema S, Schweizer S. Emotion-regulation strategies across psychopathology: a meta-analytic review. Clin Psychol Rev. 2010;30(2):217-237.

5. Berking M, Wupperman P. Emotion regulation and mental health: recent findings, current challenges, and future directions. Curr Opin Psychiatry. 2012;25(2):128-134.

6. Gross JJ, John OP. Individual differences in two emotion regulation processes: implications for affect, relationships, and well-being. J Pers Soc Psychol. 2003;85(2):348-362.

7. Gross JJ. The emerging field of emotion regulation: an integrative review. Rev Gen Psychol. 1998;2(3):271-299.

8. Lazarus RS, Alfert E. Short-circuiting of threat by experimentally altering cognitive appraisal. J Abnorm Psychol. 1964;69:195-205.

9. Richards JM, Gross JJ. Emotion regulation and memory: the cognitive costs of keeping one's cool. J Pers Soc Psychol. 2000;79(3): 410-424.

10. Butler EA, Egloff B, Wilhelm FH, Smith NC, Erickson EA, Gross JJ. The social consequences of expressive suppression. Emotion. 2003; 3(1):48-67.

11. Sheppes G, Scheibe S, Suri G, Gross JJ. Emotion-regulation choice. Psychol Sci. 2011;22:1391-1396.

12. Sheppes G, Levin, Z. Emotion regulation choice: selecting between cognitive regulation strategies to control emotion. Front Hum Neurosci. 2013;7:179.

13. Campbell-Sills L, Barlow DH, Brown TA, Hofmann SG. Effects of suppression and acceptance on emotional responses of individuals with anxiety and mood disorders. Behav Res Ther. 2006;44(9): $1251-1263$.

14. Garnefski N, Kraaij V. The Cognitive Emotion Regulation Questionnaire: psychometric features and prospective relationships with depression and anxiety in adults. Eur J Psychol Assess. 2007; 23(3):141-149.

15. Moore SA, Zoellner LA, Mollenholt N. Are expressive suppression and cognitive reappraisal associated with stress-related symptoms? Behav Res Ther. 2008;46(9):993-1000.

16. Joormann J, Gotlib IH. Emotion regulation in depression: relation to cognitive inhibition. Cogn Emot. 2010;24(2):281-298.

17. Beevers CG, Meyer B. Thought suppression and depression risk. Cognition and Emotion. 2004;18:859-867.

18. Wenzlaff RM, Luxton DD. The role of thought suppression in depressive rumination. Cognit Ther Res. 2003;27(3):293-308.

19. Matheson K, Anisman H. Systems of coping associated with dysphoria, anxiety and depressive illness: a multivariate profile perspective. Stress. 2003;6(3):223-234. 
20. Kashdan TB, Breen WL. Materialism and diminished well-being: experiential avoidance as a mediating mechanism. J Soc Clin Psychol. 2007;26(5):521-539.

21. Kraaij V, Pruymboom E, Garnefski N. Cognitive coping and depressive symptoms in the elderly: a longitudinal study. Aging Ment Health. 2002;6(3):275-281.

22. Berking M, Wupperman P, Reichardt A, Pejic T, Dippel A, Znoj H. Emotion-regulation skills as a treatment target in psychotherapy. Behav Res Ther. 2008;46(11):1230-1237.

23. World Health Organization. The ICD-10 Classification of Mental and Behavioral Disorders: Clinical Descriptions and Diagnostic Guidelines. 10th ed. Geneva: World Health Organization; 1992.

24. Hiller W, Zaudiga M, Mombour W. ICD International Diagnostic Checklists for ICD-10 and DSM-IV. Goettingen: Hogrefe and Huber Pub; 1999.

25. Hautzinger M. [Depression]. Göttingen: Hogrefe; 1998. German.

26. Beck AT, Steer RA. Beck Depression Inventory. San Antonio: The Psychological Corporation Inc.; 1987.

27. Hautzinger M, Bailer M, Hellgard W. Beck-Depressions-Inventar: Testhandbuch. 2nd ed. Bern: Hans Huber; 1995.

28. Blanchard-Fields F, Stein R, Watson TL. Age differences in emotion-regulation strategies in handling everyday problems. J Gerontol B Psychol Sci Soc Sci. 2004;59(6):261-269.

29. Mauchly JW. Significance test for sphericity of a normal n-variate distribution. The Annals of Mathematical Statistics. 1940;11:204-209.

30. Greenhouse SW, Geisser, S. On methods in the analysis of profile data. Psychometrika. 1959;24:95-112.

31. Cohen J. A power primer. Psychol Bull. 1992;11(1)2:155-159.
32. Cohen J. Statistical Power for the Behavioural Science. 2nd ed. Hillsdale, NJ: Erlbaum; 1988.

33. Fresco DM, Moore MT, van Dulmen MH, et al. Initial psychometric properties of the experiences questionnaire: validation of a self-report measure of decentering. Behav Ther. 2007;38(3):234-246.

34. Fresco DM, Segal ZV, Buis T, Kennedy S. Relationship of post treatment decentering and cognitive reactivity to relapse in major depression. $J$ Consult Clin Psychol. 2007;75(3):447-455.

35. Watkins E, Teasdale JD, Williams RM. Decentering and distraction reduce over general autobiographical memory in depression. Psychol Med. 2000;30(4):911-920.

36. Safran J, Segal Z. Interpersonal Process in Cognitive Therapy. New York: Basic Books; 1990.

37. Kuyken W, Watkins E, Holden E, et al. How does mindfulness-based cognitive therapy work? Behav Res Ther. 2010;48:1105-1112.

38. Ma SH, Teasdale JD. Mindfulness-based cognitive therapy for depression: replication and exploration of differential relapse prevention effects. J Consult Clin Psychol. 2004;72:31-40.

39. Teasdale JD, Moore RG, Hayhurst H, Pope M, Williams S, Segal ZV. Metacognitive awareness and prevention of relapse in depression: empirical evidence. J Consult Clin Psychol. 2002;70(2):275-287.

40. Corcoran KM, Segal ZV. Metacognition in depressive and anxiety disorders: current directions. Int J Cogn Ther. 2008;1(1):33-44.

41. Gratz KL, Roemer L. Multidimensional assessment of emotion regulation and dysregulation: Development, factor structure, and initial validation of the difficulties in emotion regulation scale. J Psychopathol Behav. 2004;26:41-54.
Psychology Research and Behavior Management

\section{Publish your work in this journal}

Psychology Research and Behavior Management is an international, peerreviewed, open access journal focusing on the science of psychology and its application in behavior management to develop improved outcomes in the clinical, educational, sports and business arenas. Specific topics covered include: Neuroscience, memory \& decision making; Behavior

\section{Dovepress}

modification \& management; Clinical applications; Business \& sports performance management; Social and developmental studies; Animal studies. The manuscript management system is completely online and includes a quick and fair peer-review system. Visit http://www.dovepress. com/testimonials.php to read real quotes from published authors. 